\title{
Some properties of a linear operator involving generalized Mittag-Leffler function
}

\author{
Basem Aref Frasin, Tariq Al-Hawary and Feras Yousef
}

\begin{abstract}
This paper introduces a new class $T_{\alpha, \beta, k}^{\gamma}(\eta)$ of analytic functions which is defined by means of a linear operator involving generalized Mittag-Leffler function $\mathcal{H}_{\alpha, \beta, k}^{\gamma}(f)$. The results investigated in this paper include, an inclusion relation for functions in the class $T_{\alpha, \beta, k}^{\gamma}(\eta)$ and also some subordination results of the linear operator $\mathcal{H}_{\alpha, \beta, k}^{\gamma}(f)$. Several consequences of our results are also pointed out.
\end{abstract}

Mathematics Subject Classification (2010): 33E12, 30C45.

Keywords: Analytic functions, univalent functions, Mittag-Leffler function, differential subordination, convex function.

\section{Introduction}

Let $\mathcal{A}$ denote the class of the normalized functions of the form

$$
f(z)=z+\sum_{n=2}^{\infty} a_{n} z^{n}
$$

which are analytic in the open unit disk $\mathbb{U}=\{z \in \mathbb{C}:|z|<1\}$. Further, let $f$ and $g$ be analytic functions in $\mathbb{U}$, then we say that $f$ is subordinate to $g$, written $f \prec g$ or $f(z) \prec g(z)$, if there exists an analytic function $w$ on $\mathbb{U}$ such that $w(0)=0,|w(z)|<1$ and $f(z)=g(w(z))$ for all $z \in \mathbb{U}$. In particular, if $g$ is univalent in $\mathbb{U}$, then we have

$$
f(z) \prec g(z) \Leftrightarrow f(0)=g(0) \text { and } f(\mathbb{U}) \subset g(\mathbb{U}) .
$$

Let $E_{\alpha}(z)$ be the Mittag-Leffler function [11] defined by

$$
E_{\alpha}(z)=\sum_{n=0}^{\infty} \frac{z^{n}}{\Gamma(\alpha n+1)}, \quad(z, \alpha \in \mathbb{C} ; \operatorname{Re}(\alpha)>0) .
$$


A more general function $E_{\alpha, \beta}$ generalizing $E_{\alpha}(z)$ was introduced by Wiman [14] and defined by

$$
E_{\alpha, \beta}(z)=\sum_{n=0}^{\infty} \frac{z^{n}}{\Gamma(\alpha n+\beta)}, \quad(z, \alpha, \beta \in \mathbb{C} ; \operatorname{Re}(\alpha)>0) .
$$

Moreover, Srivastava and Tomovski [13] introduced the function $E_{\alpha, \beta}^{\gamma, k}(z)$ as

$$
E_{\alpha, \beta}^{\gamma, k}(z)=\sum_{n=0}^{\infty} \frac{(\gamma)_{n k} z^{n}}{\Gamma(\alpha n+\beta) n !},(\alpha, \beta, \gamma \in \mathbb{C} ; \operatorname{Re}(\alpha)>\max \{0, \operatorname{Re}(k)-1\} ; \operatorname{Re}(k)>0),
$$

where $(\gamma)_{n}$ is Pochhammer symbol (or the shifted factorial, since $(1)_{n}=n$ !) is given in term of the Gamma functions can be written as

$$
(\gamma)_{n}=\frac{\Gamma(\gamma+n)}{\Gamma(\gamma)}= \begin{cases}1, & \text { if } n=0 \\ \gamma(\gamma+1) \ldots(\gamma+n-1), & \text { if } n \in \mathbb{N}\end{cases}
$$

The Mittag-Leffler function arises naturally in the solution of fractional order differential and integral equations, and especially in the investigations of fractional generalization of kinetic equation, random walks, Lévy flights, super-diffusive transport and in the study of complex systems. Several properties of Mittag-Leffler function and generalized Mittag-Leffler function can be found e.g. in $[2,3,4,6,7,8,9,11,12,13]$.

In [1], Attiya defined the operator $\mathcal{H}_{\alpha, \beta, k}^{\gamma}(f): \mathcal{A} \rightarrow \mathcal{A}$ by

$$
\mathcal{H}_{\alpha, \beta, k}^{\gamma}(f)(z)=Q_{\alpha, \beta, k}^{\gamma}(z) * f(z), \quad(z \in \mathbb{U}),
$$

where

$$
\begin{aligned}
& Q_{\alpha, \beta, k}^{\gamma}(z)=\frac{\Gamma(\alpha+\beta)}{(\gamma)_{k}}\left(E_{\alpha, \beta}^{\gamma, k}(z)-\frac{1}{\Gamma(\beta)}\right), \quad(z \in \mathbb{U}), \\
& (\alpha, \beta, \gamma \in \mathbb{C} ; \operatorname{Re}(\alpha)>\max \{0, \operatorname{Re}(k)-1\} ; \operatorname{Re}(k)>0 ; \\
& \operatorname{Re}(\alpha)=0 \text { when } \operatorname{Re}(k)=1 \text { with } \beta \neq 0),
\end{aligned}
$$

and the symbol $(*)$ denotes the Hadamard product (or convolution).

We note that,

$$
\mathcal{H}_{\alpha, \beta, k}^{\gamma}(f)(z)=z+\sum_{n=2}^{\infty} \frac{\Gamma(\gamma+n k) \Gamma(\alpha+\beta)}{\Gamma(\gamma+k) \Gamma(\beta+\alpha n) n !} a_{n} z^{n}
$$

It can be easily verified from (1.4) that

$$
z\left(\mathcal{H}_{\alpha, \beta, k}^{\gamma}(f)(z)\right)^{\prime}=\left(\frac{\gamma+k}{k}\right)\left(\mathcal{H}_{\alpha, \beta, k}^{\gamma+1}(f)(z)\right)-\frac{\gamma}{k}\left(\mathcal{H}_{\alpha, \beta, k}^{\gamma}(f)(z)\right) .
$$

Also we have

$$
\mathcal{H}_{0, \beta, 1}^{1}(f)(z)=f(z), \mathcal{H}_{0, \beta, 1}^{2}(f)(z)=\frac{1}{2}\left(f(z)+z f^{\prime}(z)\right) \text { and } \mathcal{H}_{0, \beta, 1}^{0}(f)(z)=\int_{0}^{z} \frac{1}{t} f(t) d t
$$


Definition 1.1. We say that the function $f \in \mathcal{A}$ is in the class $T_{\alpha, \beta, k}^{\gamma}(\eta), \eta \in[0,1)$, if $f$ satisfies the condition

$$
\operatorname{Re}\left[\mathcal{H}_{\alpha, \beta, k}^{\gamma}(f)(z)\right]^{\prime}>\eta, \quad(z \in \mathbb{U}) .
$$

The object of this paper is to investigate an inclusion relation for functions in the class $T_{\alpha, \beta, k}^{\gamma}(\eta)$ and obtain some subordination results for functions defined by the linear operator $\mathcal{H}_{\alpha, \beta, k}^{\gamma}(f)$. Several consequences of our results are also discussed.

The following results will be required in our investigation.

Lemma 1.2. ([5]) If $p(z)=1+p_{1} z+p_{2} z^{2}+\cdots$ is analytic in $\mathbb{U}$ and $h(z)$ is convex function in $\mathbb{U}$ with $h(0)=1$ and $\mu$ is a complex constant such that $\operatorname{Re} \mu>0$, then

$$
p(z)+\frac{z p^{\prime}(z)}{\mu} \prec h(z),
$$

implies

$$
p(z) \prec q(z) \prec h(z),
$$

where

$$
q(z)=\frac{\mu}{z^{\mu}} \int_{0}^{z} h(t) t^{\mu-1} d t
$$

and $q(z)$ is the best dominant.

Lemma 1.3. ([10]) Let $q$ be a convex function in $\mathbb{U}$ and let

$$
h(z)=q(z)+\alpha z q^{\prime}(z),
$$

where $\alpha>0$. If

$$
p(z)=q(0)+p_{1} z+\cdots
$$

and

$$
p(z)+\alpha z p^{\prime}(z) \prec h(z),
$$

then

$$
p(z) \prec q(z),
$$

and this result is sharp.

\section{Inclusion relation}

We begin by showing the following inclusion relation.

Theorem 2.1. If $\eta \in[0,1)$, then

$$
T_{\alpha, \beta, k}^{\gamma+1}(\eta) \subset T_{\alpha, \beta, k}^{\gamma}(\delta),
$$

where

$$
\delta=\delta(\eta, \gamma, k)=2 \eta-1+\frac{2(1-\eta)(\gamma+k)}{k} \mathbf{B}\left(\frac{\gamma+k}{k}\right),
$$


$\mathbf{B}$ being the Beta function defined by

$$
\mathbf{B}(x)=\int_{0}^{1} \frac{t^{x-1}}{t+1} d t .
$$

Proof. Let $f \in T_{\alpha, \beta, k}^{\gamma+1}(\eta)$ and define the function $p(z)$ by

$$
p(z)=\left(\mathcal{H}_{\alpha, \beta, k}^{\gamma}(f)(z)\right)^{\prime} .
$$

Making use the identity (1.5), we get

$$
\left(\mathcal{H}_{\alpha, \beta, k}^{\gamma+1}(f)(z)\right)^{\prime}=p(z)+\frac{k}{\gamma+k} z p^{\prime}(z), \quad(z \in \mathbb{U}) .
$$

Since $f \in T_{\alpha, \beta, k}^{\gamma+1}(\eta)$, from Definition 1.1 we have

$$
\operatorname{Re}\left(\mathcal{H}_{\alpha, \beta, k}^{\gamma+1}(f)(z)\right)^{\prime}>\eta, \quad(z \in \mathbb{U}) .
$$

Using (2.5) we get

$$
\operatorname{Re}\left(p(z)+\frac{k}{\gamma+k} z p^{\prime}(z)\right)>\eta
$$

which is equivalent to

$$
p(z)+\frac{k}{\gamma+k} z p^{\prime}(z) \prec \frac{1+(2 \eta-1) z}{1+z} \equiv h(z) .
$$

By using Lemma 1.2, with $\mu=\frac{\gamma+k}{k}$ we have

$$
p(z) \prec q(z) \prec h(z),
$$

where

$$
\begin{aligned}
q(z) & =\frac{\gamma+k}{k z^{\frac{\gamma+k}{k}}} \int_{0}^{z} \frac{1+(2 \eta-1) t}{1+t} t^{\frac{\gamma+k}{k}}-1 d t \\
& =\frac{\gamma+k}{k z^{\frac{\gamma+k}{k}}} \int_{0}^{z}[2 \eta-1+2(1-\eta)] \frac{1}{1+t} t^{\frac{\gamma+k}{k}-1} d t \\
& =\frac{\gamma+k}{k z^{\frac{\gamma+k}{k}}} \int_{0}^{z}(2 \eta-1) t^{\frac{\gamma+k}{k}-1} d t+\frac{2(1-\eta)(\gamma+k)}{k z^{\frac{\gamma+k}{k}}} \int_{0}^{z} \frac{t^{\frac{\gamma+k}{k}}-1}{1+t} d t \\
& =2 \eta-1+\frac{2(1-\eta)(\gamma+k)}{k z^{\frac{\gamma+k}{k}}} \int_{0}^{z} \frac{t^{\frac{\gamma+k}{k}-1}}{1+t} d t
\end{aligned}
$$

The function $q$ is convex and is the best dominant.

Since $p(z) \prec q(z)$, we get

$$
\operatorname{Re}\left[\mathcal{H}_{\alpha, \beta, k}^{\gamma}(f)(z)\right]^{\prime}>q(1)=\delta
$$


where

$$
\delta=\delta(\eta, \gamma, k)=2 \eta-1+\frac{2(1-\eta)(\gamma+k)}{k} \mathbf{B}\left(\frac{\gamma+k}{k}\right) .
$$

From (2.6) we deduce that $T_{\alpha, \beta, k}^{\gamma+1}(\eta) \subset T_{\alpha, \beta, k}^{\gamma}(\delta)$.

\section{Subordination results}

With the help of Lemma 1.3, we obtain the following result.

Theorem 3.1. Let $q(z)$ be convex univalent in $\mathbb{U}$ with $q(0)=1$ and let $h$ be a function such that

$$
h(z)=q(z)+\frac{k}{\gamma+k} z q^{\prime}(z) .
$$

If $f \in \mathcal{A}$ and verifies the differential subordination

$$
\left(\mathcal{H}_{\alpha, \beta, k}^{\gamma+1}(f)(z)\right)^{\prime} \prec h(z)
$$

then

$$
\left(\mathcal{H}_{\alpha, \beta, k}^{\gamma}(f)(z)\right)^{\prime} \prec q(z)
$$

and the result is sharp.

Proof. From (2.5) and (3.2) we obtain

$$
p(z)+\frac{k}{\gamma+k} z p^{\prime}(z) \prec q(z)+\frac{k}{\gamma+k} z q^{\prime}(z) \equiv h(z),
$$

then, by using Lemma 1.3 we get

$$
p(z) \prec q(z),
$$

that is,

$$
\left(\mathcal{H}_{\alpha, \beta, k}^{\gamma}(f)(z)\right)^{\prime} \prec q(z), \quad(z \in \mathbb{U}),
$$

and this result is sharp.

Theorem 3.2. Let $h \in \mathcal{A}$ with $h(0)=1$ and $h^{\prime}(0) \neq 0$, which verifies the inequality

$$
\operatorname{Re}\left[1+\frac{z h^{\prime \prime}(z)}{h^{\prime}(z)}\right]>-\frac{1}{2}, \quad(z \in \mathbb{U}) \text {. }
$$

If $f \in \mathcal{A}$ and verifies the differential subordination

$$
\left(\mathcal{H}_{\alpha, \beta, k}^{\gamma+1}(f)(z)\right)^{\prime} \prec h(z)
$$

then

$$
\left(\mathcal{H}_{\alpha, \beta, k}^{\gamma}(f)(z)\right)^{\prime} \prec q(z),
$$

where

$$
q(z)=\frac{\gamma+k}{k z^{\frac{\gamma+k}{k}}} \int_{0}^{z} h(t) t^{\frac{\gamma+k}{k}-1} d t .
$$

The function $q$ is convex and is the best dominant. 
Proof. If we let

$$
p(z)=\left(\mathcal{H}_{\alpha, \beta, k}^{\gamma}(f)(z)\right)^{\prime},
$$

and using the identity (1.5), we obtain

$$
\left(\mathcal{H}_{\alpha, \beta, k}^{\gamma+1}(f)(z)\right)^{\prime}=p(z)+\frac{k}{\gamma+k} z p^{\prime}(z), \quad(z \in \mathbb{U}) .
$$

Therefore, (3.5) becomes

$$
p(z)+\frac{k}{\gamma+k} z p^{\prime}(z) \prec h(z) .
$$

By using Lemma 1.2, we get

$$
p(z) \prec q(z)=\frac{\gamma+k}{k z^{\frac{\gamma+k}{k}}} \int_{0}^{z} h(t) t^{\frac{\gamma+k}{k}-1} d t,
$$

that is,

$$
\left(\mathcal{H}_{\alpha, \beta, k}^{\gamma}(f)(z)\right)^{\prime} \prec q(z), \quad(z \in \mathbb{U}) .
$$

Theorem 3.3. Let $q(z)$ be convex univalent in $\mathbb{U}$ with $q(0)=1$. And let $h$ be a function such that

$$
h(z)=q(z)+z q^{\prime}(z), \quad(z \in \mathbb{U}) .
$$

If $f \in A$ and verifies the differential subordination

$$
\left(\mathcal{H}_{\alpha, \beta, k}^{\gamma}(f)(z)\right)^{\prime} \prec h(z)
$$

then

$$
\frac{\mathcal{H}_{\alpha, \beta, k}^{\gamma}(f)(z)}{z} \prec q(z),
$$

and the result is sharp.

Proof. Let the function $p(z)$ be defined by

$$
p(z)=\frac{\mathcal{H}_{\alpha, \beta, k}^{\gamma}(f)(z)}{z} .
$$

Then, by differentiating (3.10), we get

$$
\left(\mathcal{H}_{\alpha, \beta, k}^{\gamma}(f)(z)\right)^{\prime}=p(z)+z p^{\prime}(z), \quad(z \in \mathbb{U}) .
$$

Thus (3.8) becomes

$$
p(z)+z p^{\prime}(z) \prec q(z)+z q^{\prime}(z) \equiv h(z),
$$

and from Lemma 1.3 we get (3.9).

Theorem 3.4. Let $h \in \mathcal{A}$ with $h(0)=1$ and $h^{\prime}(0) \neq 0$, which verifies the inequality (3.4). If $f \in \mathcal{A}$ and verifies the differential subordination

$$
\left(\mathcal{H}_{\alpha, \beta, k}^{\gamma}(f)(z)\right)^{\prime} \prec h(z), \quad(z \in \mathbb{U})
$$


then

$$
\frac{\mathcal{H}_{\alpha, \beta, k}^{\gamma}(f)(z)}{z} \prec q(z), \quad(z \in \mathbb{U}, z \neq 0),
$$

where

$$
q(z)=\frac{1}{z} \int_{0}^{z} h(t) d t
$$

The function $q$ is convex and is the best dominant.

Proof. Let the function $p(z)$ be defined as in (3.10). Then from (3.11) and (3.12),we have

$$
p(z)+z p^{\prime}(z) \prec h(z) .
$$

By using Lemma 1.2, we get

$$
p(z) \prec q(z)=\frac{1}{z} \int_{0}^{z} h(t) d t,
$$

and $q$ is convex and is the best dominant.

If we set $\gamma=1, \alpha=0$ and $k=1$, in Theorems 3.1-3.4, we immediately have the following special cases.

Corollary 3.5. Let $q(z)$ be convex univalent in $\mathbb{U}$ with $q(0)=1$ and let $h$ be a function such that

$$
h(z)=q(z)+\frac{1}{2} z q^{\prime}(z)
$$

If $f \in \mathcal{A}$ and verifies the differential subordination

$$
f^{\prime}(z)+\frac{1}{2} z f^{\prime \prime}(z) \prec h(z),
$$

then

$$
f^{\prime}(z) \prec q(z)
$$

and the result is sharp.

Corollary 3.6. Let $h \in \mathcal{A}$ with $h(0)=1$ and $h^{\prime}(0) \neq 0$, which verifies the inequality (3.4). If $f \in \mathcal{A}$ and verifies the differential subordination

$$
f^{\prime}(z)+\frac{1}{2} z f^{\prime \prime}(z) \prec h(z)
$$

then

$$
f^{\prime}(z) \prec q(z)
$$

where

$$
q(z)=\frac{2}{z^{2}} \int_{0}^{z} h(t) t d t .
$$

The function $q$ is convex and is the best dominant. 
Corollary 3.7. Let $q(z)$ be convex univalent in $\mathbb{U}$ with $q(0)=1$ and let $h$ be a function such that

$$
h(z)=q(z)+z q^{\prime}(z), \quad(z \in \mathbb{U}) .
$$

If $f \in \mathcal{A}$ and verifies the differential subordination

$$
f^{\prime}(z) \prec h(z),
$$

then

$$
\frac{f(z)}{z} \prec q(z)
$$

and the result is sharp.

Corollary 3.8. Let $h \in \mathcal{A}$ with $h(0)=1$ and $h^{\prime}(0) \neq 0$, which verifies the inequality (3.4). If $f \in \mathcal{A}$ and verifies the differential subordination

$$
f^{\prime}(z) \prec h(z), \quad(z \in \mathbb{U}),
$$

then

$$
\frac{f(z)}{z} \prec q(z), \quad(z \in \mathbb{U}, z \neq 0),
$$

where

$$
q(z)=\frac{1}{z} \int_{0}^{z} h(t) d t .
$$

The function $q$ is convex and is the best dominant.

Acknowledgements. The authors would like to thank the referee for his helpful comments and suggestions.

\section{References}

[1] Attiya, A.A., Some applications of Mittag-Leffler function in the unit disk, Filomat, 30(2016), no. 7, 2075-2081.

[2] Bansal, D., Prajapat, J.K., Certain geometric properties of the Mittag-Leffler functions, Complex Var. Elliptic Equ., 61(2016), no. 3, 338-350.

[3] Frasin, B.A., An application of an operator associated with generalized Mittag-Leffler function, Konuralp Journal of Mathematics, 7(2019), no. 1, 199-202.

[4] Garg, M., Manohar, P., Kalla, S.L., A Mittag-Leffler-type function of two variables, Integral Transforms Spec. Funct., 24(2013), no. 11, 934-944.

[5] Hallenbeck, D.J., Ruscheweyh, S., Subordination by convex functions, Proc. Am. Math. Soc., 52(1975), 191-195.

[6] Kiryakova, V., Generalized Fractional Calculus and Applications, Pitman Research Notes in Mathematics Series, 301. Longman Scientific \& Technical, Harlow; copublished in the United States with John Wiley \& Sons, Inc., New York, 1994.

[7] Kiryakova, V., Multiple (multiindex) Mittag-Leffler functions and relations to generalized fractional calculus. Higher transcendental functions and their applications, J. Comput. Appl. Math., 118(2000), no. 1-2, 241-259.

[8] Kiryakova, V., The multi-index Mittag-Leffler functions as an important class of special functions of fractional calculus, Comput. Math. Appl., 59(2010), no. 5, 1885-1895. 
[9] Mainardia, F., Gorenflo, R., On Mittag-Leffler-type functions in fractional evolution processes. Higher transcendental functions and their applications, J. Comput. Appl. Math., 118(2000), no. 1-2, 283-299.

[10] Miller, S.S., Mocanu, P.T., Second order differential inequalities in the complex plane, J. Math. An. Appl., 65(1978), 289-305.

[11] Mittag-Leffler, G.M., Sur la nouvelle fonction E(x), C.R. Acad. Sci., Paris, 137(1903), 554-558.

[12] Srivastava, H.M., Frasin, B.A., Pescar, V., Univalence of integral operators involving Mittag-Leffler functions, Appl. Math. Inf. Sci., 11(2017), no. 3, 635-641.

[13] Srivastava, H.M., Tomovski, Z., Fractional calculus with an integral operator containing a generalized Mittag-Leffler function in the kernel, Appl. Math. Comp., 211(2009), 198210.

[14] Wiman, A., Über den Fundamental satz in der Theorie der Funcktionen E(x), Acta Math., 29(1905), 191-201.

Basem Aref Frasin

Al al-Bayt University, Department of Mathematics,

25113 Mafraq, Jordan

e-mail: bafrasin@yahoo.com

Tariq Al-Hawary

Al-Balqa Applied University, Ajloun College,

Department of Applied Science,

26816 Ajloun, Jordan

e-mail: tariq_amh@bau.edu.jo

Feras Yousef

University of Jordan, Department of Mathematics,

11942 Amman, Jordan

e-mail: fyousef@ju.edu.jo 